Document downloaded from:

http://hdl.handle.net/10251/36266

This paper must be cited as:

Aguilar, J.; Al Samarai, I.; Albert, A.; André, M.; Anghinolfi, M.; Anton, G.; Anvar, S.... (2011). Time calibration of the ANTARES neutrino telescope. Astroparticle Physics. 34(7):539-549. doi:10.1016/j.astropartphys.2010.12.004

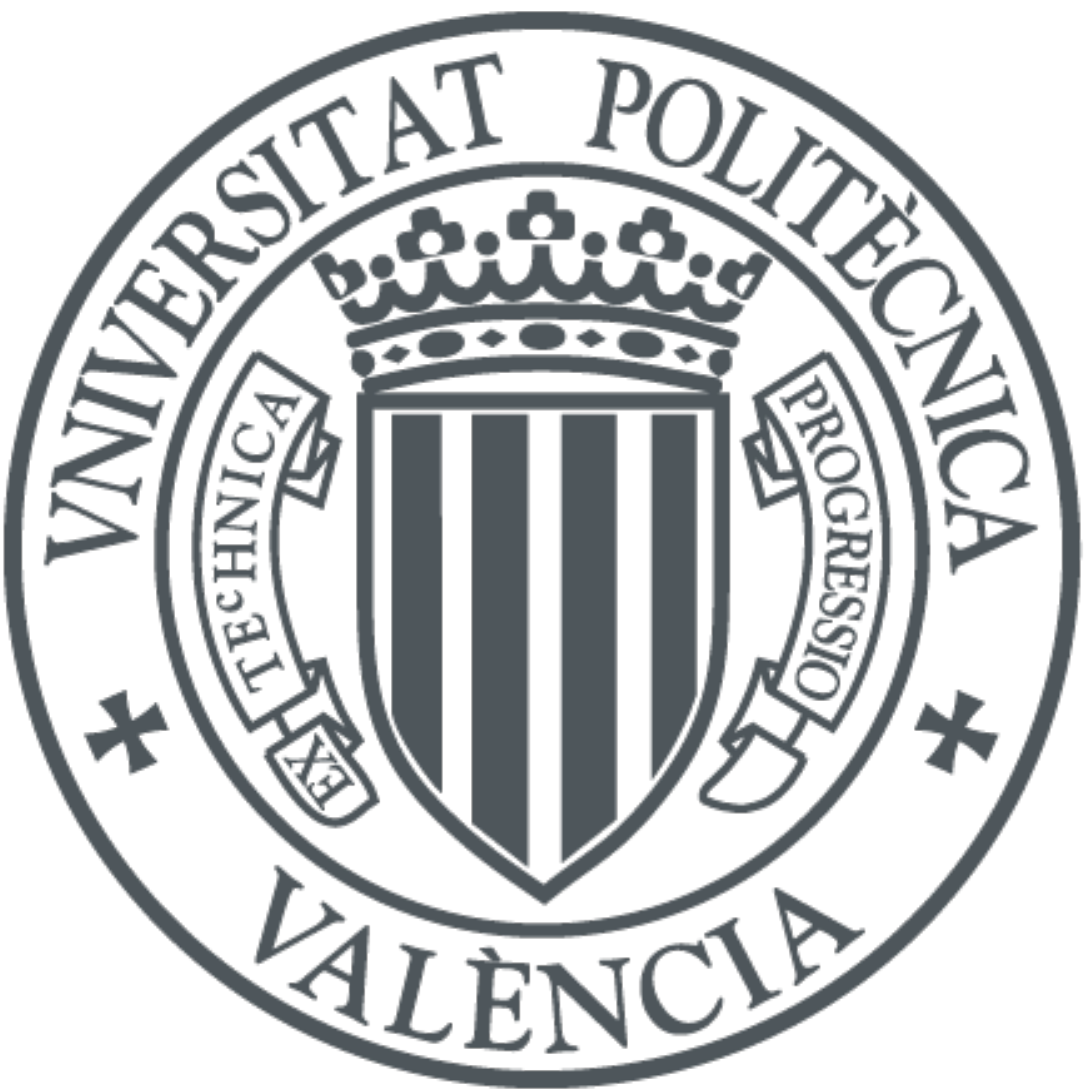

The final publication is available at

http://dx.doi.org/10.1016/j.astropartphys.2010.12.004

Copyright Elsevier

Additional Information 


\section{Time Calibration of the ANTARES Neutrino Telescope}

\section{The ANTARES Collaboration}

J.A. Aguilar ${ }^{\text {a }}$, I. Al Samarai ${ }^{\text {b }}$, A. Albert ${ }^{\mathrm{c}}$, M. Andréd, M. Anghinolfi ${ }^{\mathrm{e}}$, G. Anton ${ }^{\text {f }}$, S. Anvar ${ }^{\mathrm{g}}$, M. Ardid ${ }^{\text {h}}$, A.C. Assis Jesus ${ }^{\mathrm{i}}$, T. Astraatmadja ${ }^{\mathrm{i}}$, J.J. Aubert ${ }^{\mathrm{b}}$, R. Auer ${ }^{\mathrm{f}}$, B. Baret $^{\mathrm{j}}$, S. Basa ${ }^{\mathrm{k}}$, M. Bazzotti ${ }^{\ell, \mathrm{m}}$, V. Bertin ${ }^{\mathrm{b}}$,

S. Biagi ${ }^{\ell, m}$, C. Bigongiari ${ }^{a}$, M. Bou-Cabo ${ }^{\text {h }}$, M.C. Bouwhuis ${ }^{\mathrm{i}}$,

A. M. Brown ${ }^{\text {b }}$, J. Brunner ${ }^{\mathrm{b}}{ }^{2}$, J. Busto ${ }^{\mathrm{b}}$, F. Camarena ${ }^{\mathrm{h}}$, A. Capone ${ }^{\mathrm{n}, \mathrm{o}}$, C.Cârloganu $^{\mathrm{p}}$, G. Carminati ${ }^{\ell, m}$, J. Carr $^{\mathrm{b}}$, S. Cecchini ${ }^{\mathrm{m}, \mathrm{q}}$, Ph. Charvis ${ }^{\mathrm{r}}$, T. Chiarusi ${ }^{\mathrm{m}}$, M. Circella ${ }^{\mathrm{s}}, \mathrm{H}$. Costantini ${ }^{\mathrm{e}}, \mathrm{N}_{\text {. Cottini }}{ }^{\mathrm{t}}, \mathrm{P}$. Coyle ${ }^{\mathrm{b}}$,

C. Curtil ${ }^{\text {b }}$, M.P. Decowski ${ }^{i}$, I. Dekeyser ${ }^{u}$, A. Deschamps ${ }^{r}$, C. Distefano ${ }^{v}$, C. Donzaud ${ }^{\mathrm{j}, \mathrm{w}}$, D. Dornic ${ }^{\mathrm{b}, \mathrm{a}}$, D. Drouhin ${ }^{\mathrm{c}}$, T. Eberl ${ }^{\mathrm{f}}$, U. Emanuele $^{\mathrm{a}}$, J.P. Ernenwein ${ }^{b}$, S. Escoffier ${ }^{b}$, F. Fehr ${ }^{f}$, V. Flaminio ${ }^{x, y}$, U. Fritsch ${ }^{f}$,

J.L. Fuda ${ }^{\mathrm{u}}$, S. Galata ${ }^{\mathrm{b}}$, P. Gay ${ }^{\mathrm{p}}$, G. Giacomelli, m, J.P. Gómez-González ${ }^{\mathrm{a}}$, K. Graf ${ }^{f}$, G. Guillard ${ }^{\mathrm{z}}$, G. Halladjian ${ }^{\mathrm{b}}$, G. Hallewell ${ }^{\mathrm{b}}$, H. van Haren ${ }^{\text {aa }}$, A.J. Heijboer ${ }^{\mathrm{i}}$, Y. Hello ${ }^{\mathrm{r}}$, J.J. Hernández-Rey ${ }^{\mathrm{a}}$, B. Herold ${ }^{\mathrm{f}}$, J. Hößl ${ }^{\mathrm{f}}$, C.C. Hsu ${ }^{i}$, M. de Jong ${ }^{\text {i }}{ }^{1}$, M. Kadler ${ }^{\text {ab }}$, N. Kalantar-Nayestanaki ${ }^{\text {ac }}$, O. Kalekin ${ }^{f}$, A. Kappes ${ }^{f}$, U. Katz ${ }^{f}$, P. Kooijman ${ }^{\text {i,ad,ae }}$, C. Kopper ${ }^{f}$,

A. Kouchner ${ }^{j}$, V. Kulikovskiy af,e, R. Lahmann ${ }^{f}$, P. Lamare ${ }^{g}$, G. Larosa ${ }^{\text {h }}$,

D. Lefèvre ${ }^{\mathrm{u}}$, G. Lim ${ }^{\mathrm{i}, \mathrm{ae}}$, D. Lo Presti ${ }^{\mathrm{ag}}, \mathrm{H}$. Loehner ${ }^{\mathrm{ac}}$, S. Loucatos ${ }^{\mathrm{t}}$, F. Lucarelli ${ }^{\mathrm{n}, \mathrm{o}}$, S. Mangano ${ }^{\mathrm{a}}$, M. Marcelin ${ }^{\mathrm{k}}$, A. Margiotta ${ }^{\ell, \mathrm{m}}$,

J.A. Martinez-Mora ${ }^{\text {h, A. Mazure }}{ }^{\mathrm{k}}$, T. Montaruli ${ }^{\mathrm{s}, \mathrm{ah}}$, M. Morganti ${ }^{\mathrm{x}, \mathrm{y}}$, L. Moscoso ${ }^{\mathrm{t}, \mathrm{j}}$, H. Motz ${ }^{\mathrm{f}}$,C. Naumann ${ }^{\mathrm{t}}$, M. Neff ${ }^{\mathrm{f}}$, D. Palioselitis ${ }^{\mathrm{i}}$, G.E.Păvălaş ${ }^{\text {ai }}$, P. Payre ${ }^{\mathrm{b}}$, J. Petrovic ${ }^{\mathrm{i}}$, P. Piattelli v ${ }^{\text {, N. Picot-Clemente }}{ }^{\mathrm{b}}$,

C. Picq ${ }^{\mathrm{t}}$, V. Popa ${ }^{\mathrm{ai}}$, T. Pradier $^{\mathrm{z}}$, E. Presani ${ }^{\mathrm{i}}$, C. Racca ${ }^{\mathrm{c}}$, C. Reed ${ }^{\mathrm{i}}$, G. Riccobene ${ }^{v}$, C. Richardt ${ }^{f}$, M. Rujoiu ${ }^{\text {ai }}$, G.V. Russo ${ }^{\text {ag }}$, F. Salesa ${ }^{a}$, P. Sapienza ${ }^{v}$, F. Schöck ${ }^{f}$, J.P. Schuller ${ }^{t}$, R. Shanidze ${ }^{f}$, F. Simeone ${ }^{o}$, A. Spies ${ }^{\mathrm{f}}$, M. Spurio ${ }^{\ell, m}$, J.J.M. Steijger ${ }^{\mathrm{i}}$, Th. Stolarczyk ${ }^{\mathrm{t}}$, M. Taiuti ${ }^{\text {aj,e }}$, C. Tamburini ${ }^{u}$, L. Tasca $^{\mathrm{k}}, \mathrm{S}$. Toscano ${ }^{\mathrm{a}}$, B. Vallage ${ }^{\mathrm{t}}, \mathrm{V}$. Van Elewyck ${ }^{\mathrm{j}}$, G. Vannoni ${ }^{\mathrm{t}}, \mathrm{M}$. Vecchi ${ }^{\mathrm{n}, \mathrm{b}}$, P. Vernin ${ }^{\mathrm{t}}$, G. Wijnker ${ }^{\mathrm{i}}$, E. de Wolf ${ }^{\mathrm{i}, \mathrm{ae}}$, H. Yepes ${ }^{a}$, D. Zaborov ${ }^{\text {ak }}$, J.D. Zornoza ${ }^{a}{ }^{3}$, J. Zúñiga $^{a}$

1 Also at University of Leiden, the Netherlands

2 On leave at DESY, Platanenallee 6, D-15738 Zeuthen, Germany

3 Corresponding author: zornoza@ific.uv.es 
${ }^{\mathrm{a}}$ IFIC - Instituto de Física Corpuscular, Edificios Investigación de Paterna, CSIC - Universitat de València, Apdo. de Correos 22085, 46071 Valencia, Spain

${ }^{\mathrm{b}}$ CPPM - Centre de Physique des Particules de Marseille, CNRS/IN2P3 et Université de la Méditerranée, 163 Avenue de Luminy, Case 902, 13288 Marseille Cedex 9, France

${ }^{\mathrm{c}}$ GRPHE - Institut universitaire de technologie de Colmar, 34 rue du Grillenbreit BP 50568 - 68008 Colmar, France

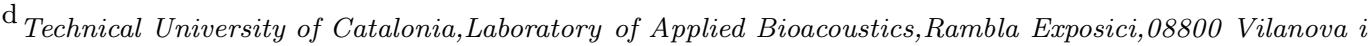
la Geltr,Barcelona, Spain

${ }^{\mathrm{e}}$ INFN - Sezione di Genova, Via Dodecaneso 33, 16146 Genova, Italy

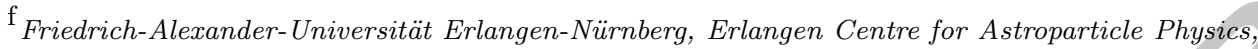
Erwin-Rommel-Str. 1, 91058 Erlangen, Germany

${ }^{\mathrm{g}}$ Direction des Sciences de la Matière - Institut de recherche sur les lois fondamentales de l'Univers Service d'Electronique des Détecteurs et d'Informatique, CEA Saclay, 91191 Gif-sur-Yvette Cedex, France

${ }^{\mathrm{h}}$ Institut d'Investigació per a la Gestió Integrada de Zones Costaneres (IGIC) - Universitat Politècnica de València. C/ Paranimf 1, 46730 Gandia, Spain.

${ }^{\mathrm{i}}$ FOM Instituut voor Subatomaire Fysica Nikhef, Science Park 105, 1098 XG Amsterdam, The Netherlands

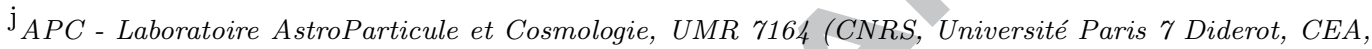
Observatoire de Paris) 10, rue Alice Domon et Léonie Duquet 75205 Paris Cedex 13, France

${ }^{\mathrm{k}}$ LAM - Laboratoire d'Astrophysique de Marseille, Pôle de l'Étoile Site de Château-Gombert, rue Frédéric Joliot-Curie 38, 13388 Marseille Cedex 13, France

${ }^{\ell}$ Dipartimento di Fisica dell'Università, Viale Berti Pichat 6/2, 40127 Bologna, Italy

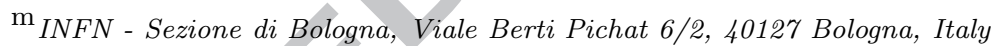

${ }^{\mathrm{n}}$ Dipartimento di Fisica dell'Università La Sapienza, P.le Aldo Moro 2, 00185 Roma, Italy

${ }^{\circ}$ INFN-Sezione di Roma, P.le Aldo Moro 2, 00185 Roma, Italy

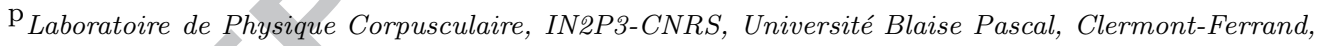
France

q INAF-IASF, via P. Gobetti 101, 40129 Bologna, Italy

${ }^{\mathrm{r}}$ Géoazur - Université de Nice Sophia-Antipolis, CNRS/INSU, IRD, Observatoire de la Côte d'Azur and Université Pierre et Marie Curie, BP 48, 06235 Villefranche-sur-mer, France

${ }^{\mathrm{s}}$ INFN - Sezione di Bari, Via E. Orabona 4, 70126 Bari, Italy

${ }^{t}$ Direction des Sciences de la Matière - Institut de recherche sur les lois fondamentales de l'Univers Service de Physique des Particules, CEA Saclay, 91191 Gif-sur-Yvette Cedex, France

${ }^{\mathrm{u}}$ COM - Centre d'Océanologie de Marseille, CNRS/INSU et Université de la Méditerranée, 163 Avenue de Luminy, Case 901, 13288 Marseille Cedex 9, France

${ }^{\mathrm{v}}$ INFN - Laboratori Nazionali del Sud (LNS), Via S. Sofia 62, 95123 Catania, Italy

${ }^{\mathrm{w}}$ Université Paris-Sud 11 - Département de Physique, 91403 Orsay Cedex, France 
${ }^{\mathrm{x}}$ Dipartimento di Fisica dell'Università, Largo B. Pontecorvo 3, 56127 Pisa, Italy

${ }^{\mathrm{y}}$ INFN - Sezione di Pisa, Largo B. Pontecorvo 3, 56127 Pisa, Italy

${ }^{\mathrm{z}}$ IPHC-Institut Pluridisciplinaire Hubert Curien - Université de Strasbourg et CNRS/IN2P3 23 rue du Loess, BP 28, 67037 Strasbourg Cedex 2, France

${ }^{\text {aa }}$ Royal Netherlands Institute for Sea Research (NIOZ), Landsdiep 4,1797 SZ 't Horntje (Texel), The Netherlands

${ }^{\mathrm{ab}}$ Dr. Remeis Sternwarte Bamberg, Sternwartstraße 7,Bamberg, Germany

${ }^{\mathrm{ac}}$ Kernfysisch Versneller Instituut (KVI), University of Groningen, Zernikelaan 25, 9747 AA Groningen, The Netherlands

ad Universiteit Utrecht, Faculteit Betawetenschappen, Princetonplein 5, 3584 CC Utrecht, The Netherlands

${ }^{\mathrm{ae}}$ Universiteit van Amsterdam, Instituut voor Hoge-Energie Fysika, Science Park 105, 1098 XG Amsterdam, The Netherlands

af Moscow State University,Skobeltsyn Institute of Nuclear Physics,Leninskie gory, 119991 Moscow, Russia

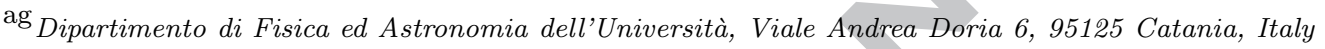

${ }^{\text {ah }}$ University of Wisconsin - Madison, 53715, WI, USA

${ }^{\text {ai }}$ Institute for Space Sciences, R-77125 Bucharest, Măgurele, Romania

${ }^{\mathrm{aj}}$ Dipartimento di Fisica dell'Università, Via Dodecaneso 33, 16146 Genova, Italy

${ }^{\mathrm{ak}}$ ITEP - Institute for Theoretical and Experimental Physics, B. Cheremushkinskaya 25, 117218 Moscow, Russia

\begin{abstract}
The ANTARES deep-sea neutrino telescope comprises a three-dimensional array of photomultipliers to detect the Cherenkov light induced by upgoing relativistic charged particles originating from neutrino interactions in the vicinity of the detector. The large scattering length of light in the deep sea facilitates an angular resolution of a few tenths of a degree for neutrino energies exceeding $10 \mathrm{TeV}$. In order to achieve this optimal performance, the time calibration procedures should ensure a relative time calibration between the photomultipliers at the level of $\sim 1 \mathrm{~ns}$. The methods developed to attain this level of precision are described.
\end{abstract}

Key words: Time calibration, Neutrino Telescopes, ANTARES 


\section{Introduction}

The ANTARES Collaboration has constructed a neutrino telescope in the Mediterranean Sea [1]. The main aim of the project is the search for highenergy neutrinos of astrophysical origin. This is achieved by the detection of Cherenkov photons induced by the passage of relativistic charged particles resulting from neutrino interactions in the material surrounding the detector, the most important channel being the charged current interactions producing muons. Other signatures, such as the cascades produced both in charged and neutral currents are also detected. These photons are detected in a large threedimensional array of 885 photomultipliers (PMTs) installed on twelve vertical lines anchored on the sea bed at a depth of $2475 \mathrm{~m}$. First physics results have been published [2-4].

Detailed simulations have shown [5] that the theoretically achievable angular resolution of the telescope (crucial for the search for cosmic point sources) in the muon channel reaches a few tenths of a degree at energies larger than 10 $\mathrm{TeV}$. This sub-degree resolution has been confirmed using data [6]. The reconstruction of the tracks is based on the probability density function (PDF) of arrival times of photons at the photomultipliers. A good timing calibration is therefore mandatory to ensure the reliability of the track reconstruction algorithms. The precision required for the relative time calibration system derives from the transit time spread (TTS) of the photomultipliers $\left(\sigma_{T T S} \sim 1.3 \mathrm{~ns}\right.$ ) and the effect of the chromatic dispersion in water $(\sim 1.5 \mathrm{~ns}$ for a typical light path of $40 \mathrm{~m})$. The addition in quadrature of these contributions leads to a best possible time resolution of $\sim 2$ ns per single photon.

The measurement of the detection times of the PMT hits is based on clock signals which are distributed from shore so that a common reference time is used in the whole apparatus, as explained in Section 3. The clock distribution system determines the propagation delays for these signals to reach the different electronics containers offshore with sub-nanosecond precision. The time measurements from individual PMTs have however to be corrected by appropriate offsets, which are determined in situ by means of a calibration system based on LED and laser devices (optical beacons) emitting light flashes at known times [7]. In order to ensure that the apparatus reaches its maximum pointing accuracy, it is necessary that the accuracy with which all such offsets are determined, in the so-called relative time calibration, remains below 1 ns, so that the resulting systematic error is significantly smaller than the intrinsic event-by-event fluctuations.

The absolute time calibration is less demanding. A precision of a few seconds is sufficient to correlate reconstructed neutrino directions with steady point sources and an accuracy of the order of milliseconds is sufficient for association 
to transient astrophysical events (GRBs, AGN flares, SGR bursts, etc.) As it will be explained later, the GPS system provides a good enough time stamping.

The structure of this article is as follows. A brief description of the ANTARES detector and its data-acquisition (DAQ) system is given in Section 2. In Section 3, the main characteristics of the master clock of the experiment, used to synchronize all detector elements, are presented. Section 4 describes the calibration of the time offsets specific to each PMT performed in the laboratory before deployment and in situ. In Section 5 independent cross-checks of the system performance are presented.

\section{The ANTARES detector}

The ANTARES detector is located in the Mediterranean Sea, about $40 \mathrm{~km}$ from Toulon, off the French coast. It consists of 885 10-inch PMTs (model R7081-20 from Hamamatsu) distributed over twelve lines, of 25 triplet PMT storeys each, anchored to the sea bottom (see Figure 1). The total length of each line is $480 \mathrm{~m}$. The lowest storey of each line is located $100 \mathrm{~m}$ above the sea bed. The distance between consecutive storeys is $14.5 \mathrm{~m}$ and the horizontal separation between lines is $60-80 \mathrm{~m}$. The PMTs [8] are coupled with optical gel to the inner surfaces of pressure-resistant glass spheres (optical modules, OMs [9]). A $\mu$-metal cage inside the OM shields the PMT from the Earth magnetic field. Each storey comprises a triplet of OMs and an electronics module (Local Control Module, LCM). Five consecutive storeys along the line are grouped into a "sector" sharing common data and clock distribution elements. One LCM of a sector (Master Local Control Module, MLCM) contains an Ethernet switch and a Dense Wavelength Division Multiplexing transceiver of a specific wavelength to establish data connection from the sector to shore along optical fibres. At the bottom of each line, a String Control Module (SCM) houses the power units and the optical (de)multiplexers. Furthermore, each line incorporates four LED Optical Beacons (OBs) [7] used for time calibration and studies of the optical properties of the sea water. In addition, two Laser Beacons are located at the bottom of two of the central lines.

The lines are connected to a junction box from where the Main Electro-Optical Cable (MEOC), of a length of $45 \mathrm{~km}$, links the detector to the shore station. The shore station houses a computer farm for data filtering and storage and the master clock. A more detailed description of the ANTARES DAQ system can be found in [10].

The signals from one PMT are processed in situ by ASICs (named Analogue Ring Samplers or ARSs [11]), located in the LCMs. Two ARSs are used in 


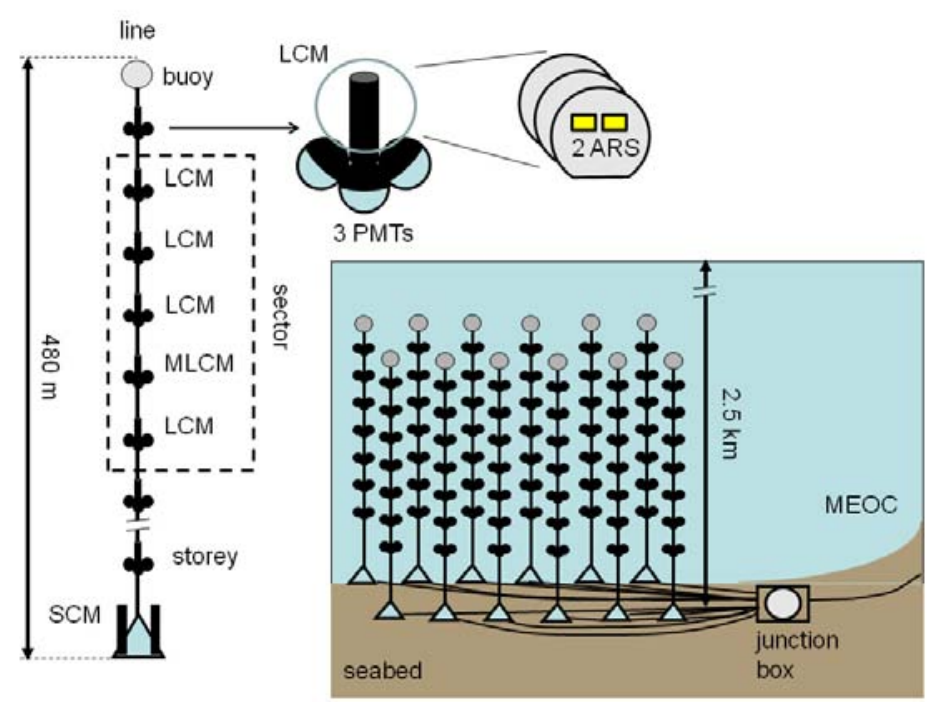

Fig. 1. Schematic view of the ANTARES detector, consisting of twelve mooring lines connected to the shore station through an electro-optical cable. Laser beacons are located at the bottom of Lines 7 and 8 (central lines). LED beacons are located in storeys $2,9,15$ and 21 (counted from bottom to top) of each line.

alternation on each OM in order to reduce the dead time of the PMT signals close in time. If an analogue PMT signal reaches an amplitude larger than a given threshold (typically 0.3 photo-electrons), its arrival time and charge are digitized by the ARS chip. The coarse signal time (time stamp) is given by a counter which is incremented by a $20 \mathrm{MHz}$ clock synchronized to signals received from shore by the clock system (see next section). A Time-to-Voltage Converter (TVC) read by an 8-bit ADC is used to measure the fine time in the 50 ns period after the time stamp. The dynamic range of the TVC is determined by recording random signals uniformly distributed in time (see Figure 2). The clock period of 50 ns covers about 150 channels of the ADC, corresponding to an average bin width of $0.3 \mathrm{~ns}$. The resulting effective time resolution is thus about $0.3 \mathrm{~ns} / \sqrt{12}$. However, differential non-linearities of the ADCs (i.e. not all bins have the same width) result in an effective precision about four times larger, which is still adequate for our requirements to keep time calibration resolution below $\sim 1$ ns.

The fact that the PMT signals are discriminated by the ARSs using a fixed amplitude threshold leads to a walk effect: a PMT signal with high amplitude crosses the threshold earlier than a coincident low amplitude signal. The difference between the threshold crossing times of pulses of different amplitudes can be of the order of a few nanoseconds. This effect can be corrected if the photo-electron pulse shape (PPS) is known: the PPS function is scaled up or down to reproduce the total charge of the hit and the corresponding thresholdcrossing time is calculated. During standard data acquisition the total charge of a PMT hit is integrated by the ARS and the information about its shape is 


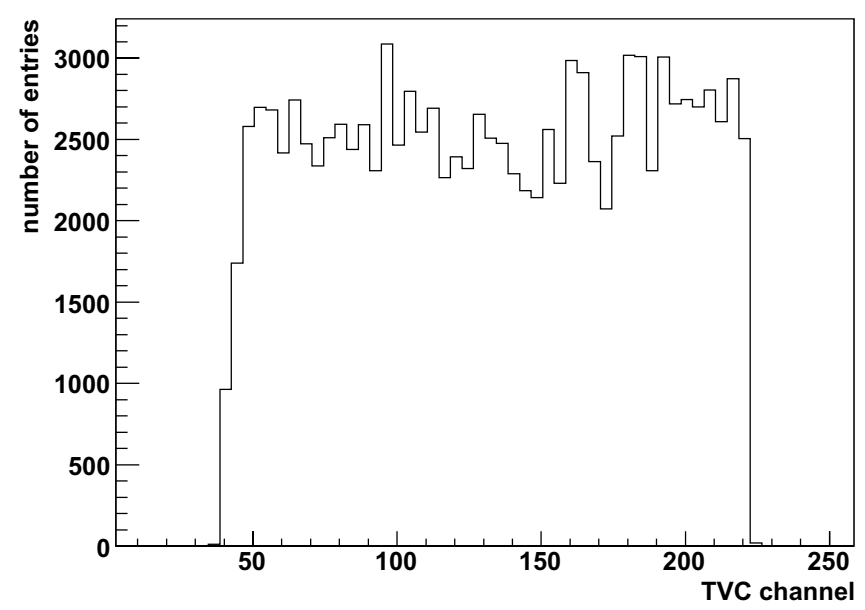

Fig. 2. TVC distribution generated from random PMT signals. The TVC values have been digitized by an ADC. The histogram has been rebinned by a factor 4 to mitigate the effect of differential non-linearities.

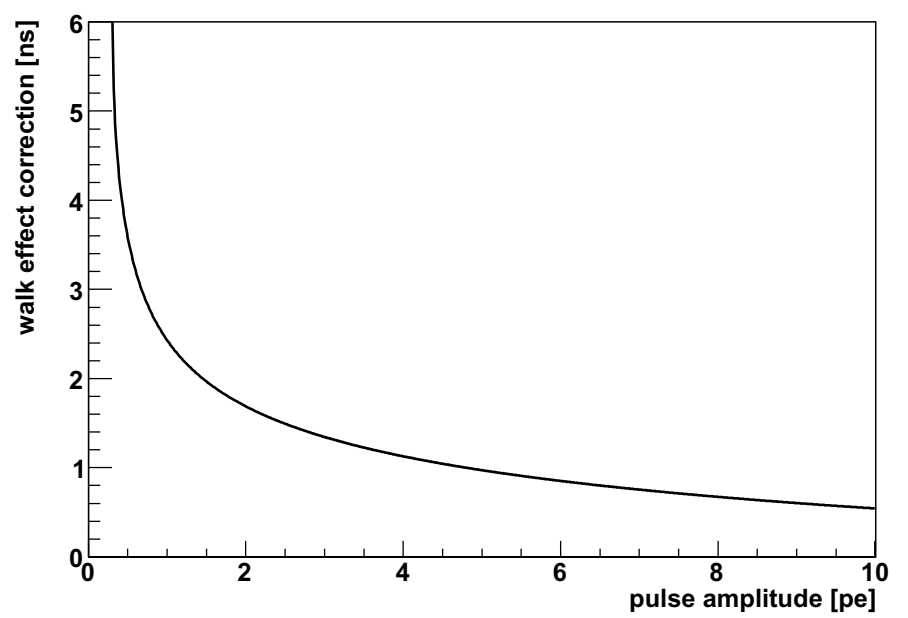

Fig. 3. Time offset due to the walk effect as a function of the PMT pulse amplitude in units of photo-electrons.

lost. However, the ARSs can be operated in a special acquisition mode which records PMT signals as waveforms sampled into 128 channels over 200 ns and digitized by an ADC. The PPS used to extract the walk correction is computed by fitting the average waveform obtained from single photo-electron events of all the ARSs. The walk effect correction as a function of the pulse amplitude is shown in Figure 3. It is systematically applied before event reconstruction. 


\section{The Clock System}

The main goal of the clock system is to provide a common signal to synchronize the readout of the OMs. It consists of a $20 \mathrm{MHz}$ generator on shore, a distribution network and a signal transceiver in each LCM. The optical clock signals received from shore are converted and decoded by an electronics board inside the LCM, and are then distributed over an electrical bus to the ARS chips.

The absolute time stamping is performed by interfacing the clock system with a dedicated electronics board to the GPS timing system that provides a time accuracy of $\sim 100 \mathrm{~ns}$ with respect to the Universal Time Coordinated, well within the requirements (a few seconds to provide the direction of the neutrino in celestial coordinates and a few milliseconds to correlate it to astrophysical phenomena).

Track reconstruction requires the knowledge of the relative arrival times of the Cherenkov photons at the PMTs and therefore only their relative time offsets.

The layout of the clock distribution network is shown in Figure 4. It is based on a bi-directional optical communication system. The clock signals are transmitted (received) between shore and the SCMs of the lines using infrared signals of $1534 \mathrm{~nm}(1549 \mathrm{~nm})$ wavelength, while within each detector line wavelengths of $1550 \mathrm{~nm}$ and $1310 \mathrm{~nm}$ are used for transmissions in the two opposite directions. In order to measure the time delays for individual storeys due to the propagation time along the cables, special clock signals are addressed to each LCM clock transceiver which responds by sending back a signal to the shore. The corresponding round-trip time is twice the propagation time to each individual LCM. During the standard operation of the telescope, the round-trip times to all the LCMs of the detector are monitored once per hour, which ensures to have at least one measurement per data taking run. An average of many such measurements provides the required precision.

Figure 5 shows an example of the time evolution of the measured round-trip time from the shore station to the SCM of a line. Clock delay variations of the order of a few hundreds of picoseconds are observed and found to be correlated with temperature variations in the onshore section of the MEOC.

Figure 6 shows the stability of the round-trip times between an LCM and the SCM of a line. In this case, the delay variations in the common part of the MEOC cable cancel out and the relative uncertainty is reduced to around 16 ps. 


\section{ACCEPTED MANUSCRIPT}

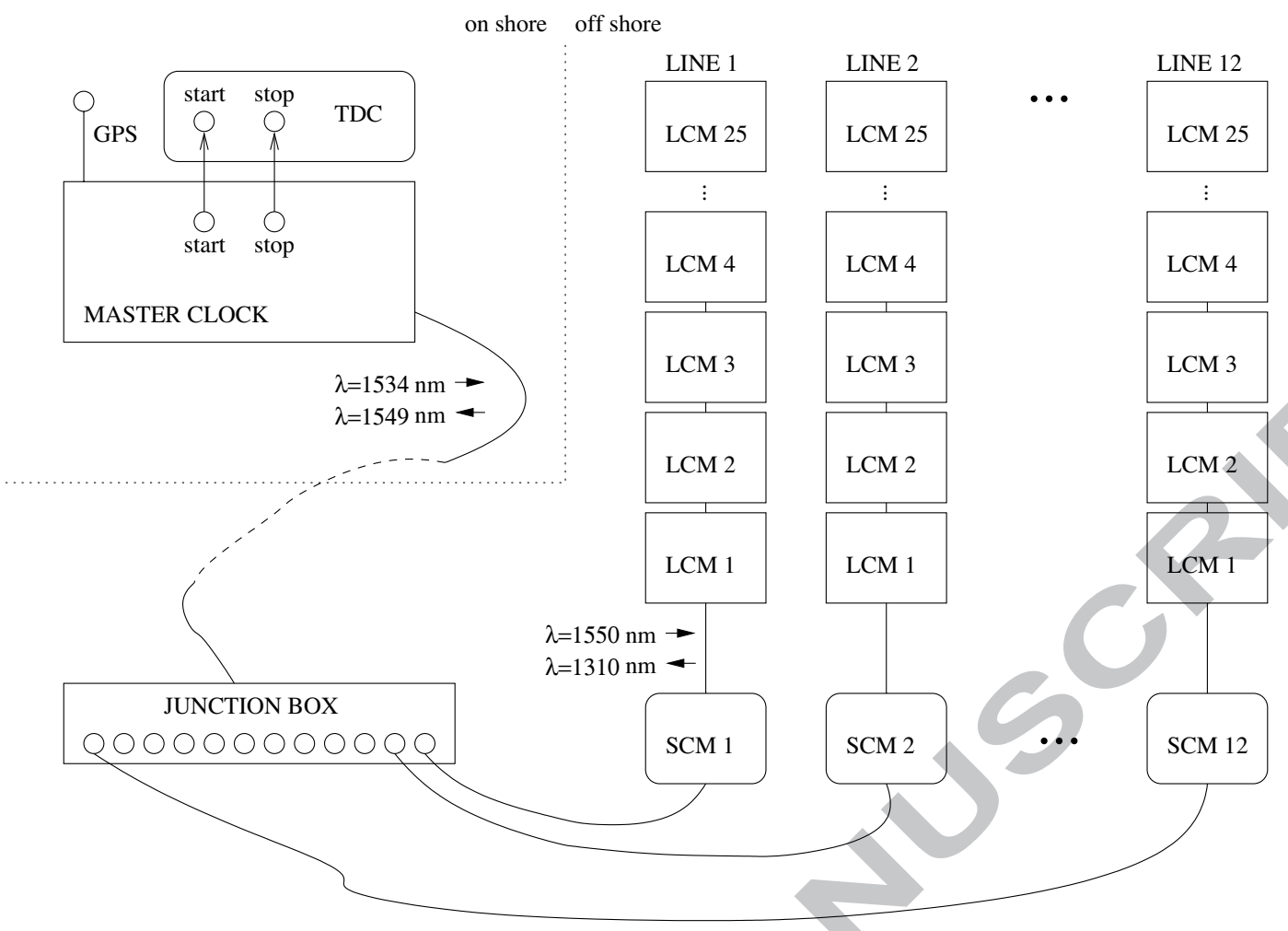

Fig. 4. Schematic view of the clock system: optical signals are sent from shore and propagated up to the junction box where they are split towards the SCMs of the different lines. From the SCM they are distributed to each LCM, where a transceiver sends the signal back to shore for calibration purposes.
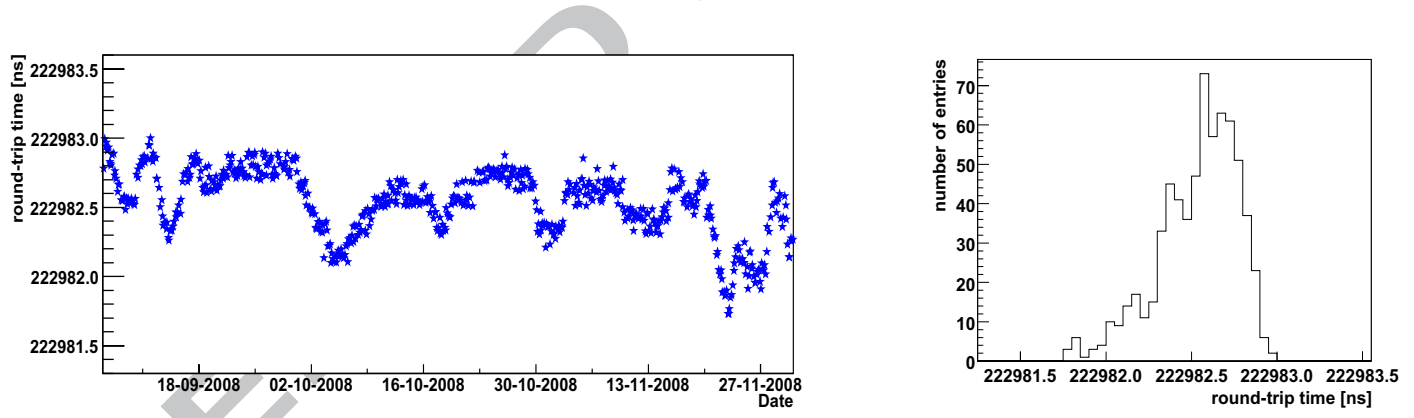

Fig. 5. Left: Time evolution of the round-trip time of a clock signal from the onshore station to an SCM for the period August-December 2008. Right: The corresponding distribution of round-trip times for the same period.

\section{OM time offset calibration}

The time elapsed between the incidence of a photon on the photocathode of the PMT and the time stamping of the associated signal in the ARS must be determined. This delay depends on the PMT signal transit time, the length of the cable linking the OM to the LCM, the front-end electronics response time and the ARS threshold. The relevant time constants are initially deter- 

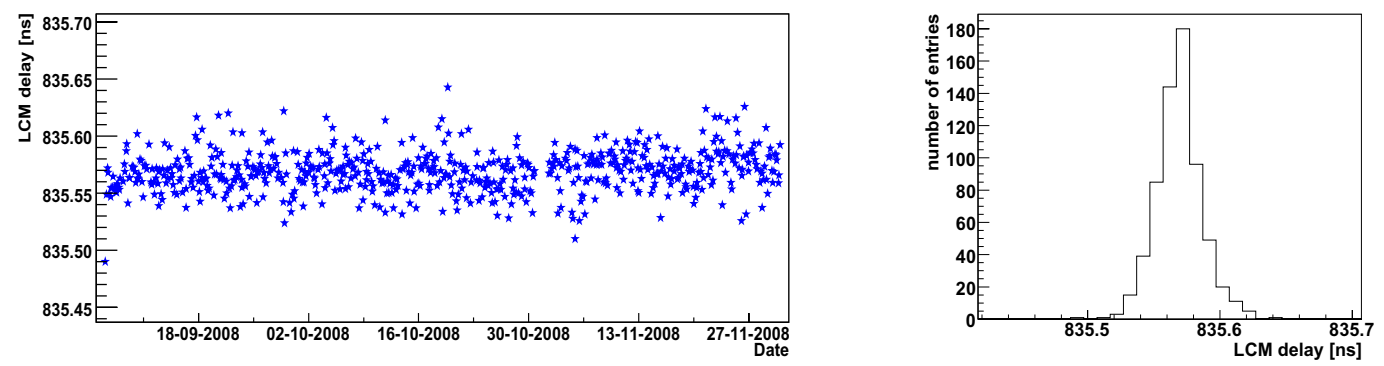

Fig. 6. Left: Time evolution of the difference in the round-trip times between the shore and an SCM and one of the LCMs. Right: Projection of these values, whose distribution has an RMS of about 16 ps.

mined with a dedicated calibration set-up in a dark room prior to deployment. These constants could change slightly after deployment due to the temperature changes, stressing of cables during transportation/deployment, etc. Morever, when the high voltage applied to the PMTs change, the time offsets change too. Using the optical beacon system, they are periodically monitored and recomputed when the lines are operated in the sea.

\subsection{Onshore calibration}

Before deployment the functionality of each line is verified and a complete calibration is performed. The onshore time calibration is carried out illuminating simultaneously groups of OMs by short laser pulses. The propagation times from SCM to LCM are known from the clock system calibration. The contribution from the cable that links the LCM to the OM, the PMT transit time and the front-end electronics delay can thus be extracted.

The time calibration is performed in batches of five storeys (a sector) in the dark room (see Figure 7). The light source used is a Q-switched, Nd-YAG laser [12] that emits intense $(\mathrm{E} \sim 1 \mu \mathrm{J})$ and short $(\mathrm{FWHM} \sim 0.8 \mathrm{~ns})$ pulses of green light $(\lambda=532 \mathrm{~nm})$ externally triggered at a frequency of $1 \mathrm{kHz}$. The light is split using a 1-to-16 optical splitter and distributed to the $15 \mathrm{OMs}$ of each sector through optical fibres. Each optical fibre is coupled to a Lambertian diffuser which spreads the laser light over the full area of the corresponding PMT photocathode. The output amplitude of the light (equivalent to about $10^{12}$ photons) is attenuated in order to produce a few tens of photoelectrons in the PMTs. The fibre lengths are adjusted such that the corresponding time differences are less than 0.3 ns. Measurements with the optical fibres swapped between OMs are made to cross-check these time differences. The time reference is provided by the laser internal photodiode. A reference LCM digitises this photodiode signal in order to synchronize the time measurements for OMs of different sectors. 


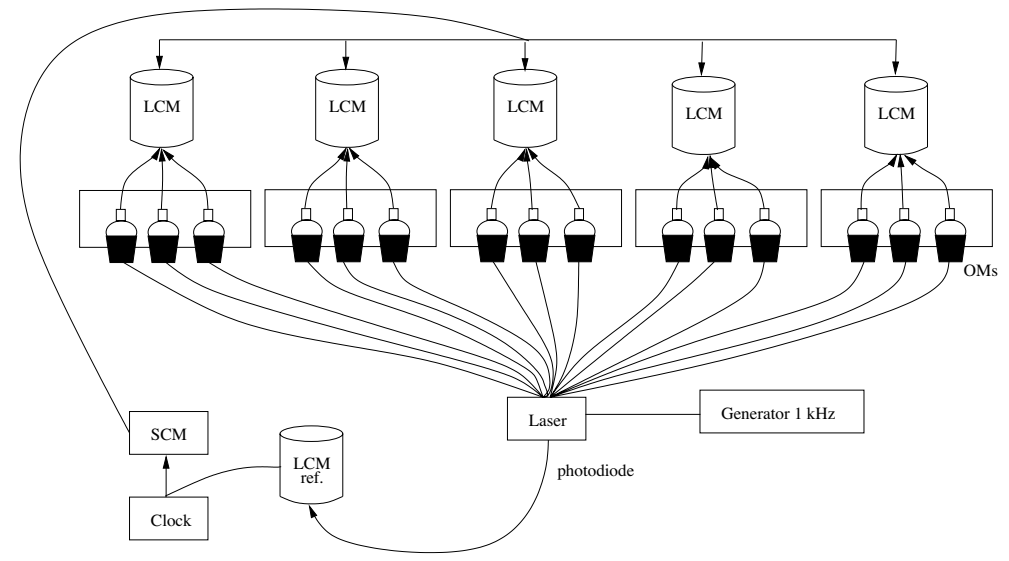

Fig. 7. Set-up for time calibration in the dark room. A laser signal is split and sent to the 15 OMs of the sector to be calibrated.

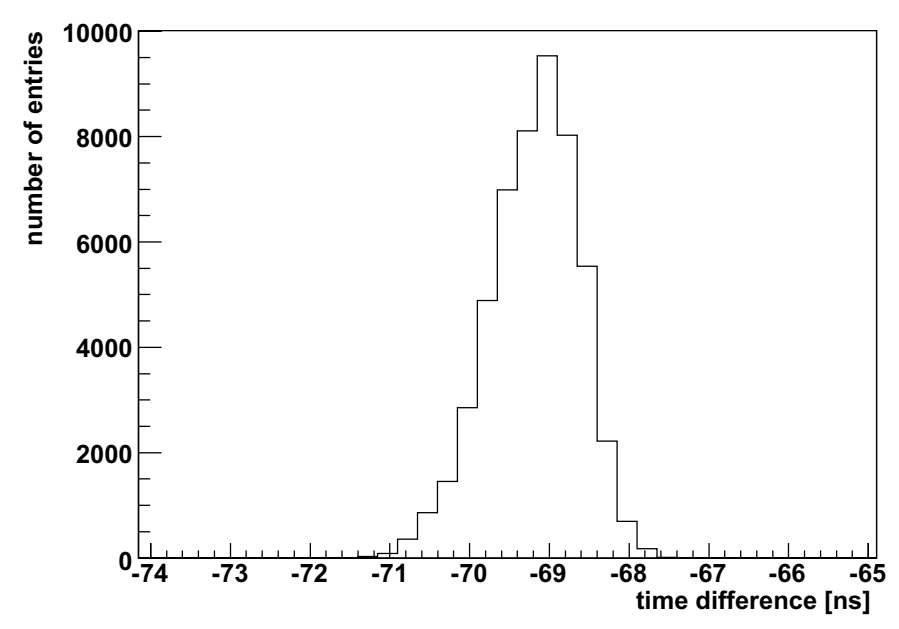

Fig. 8. An example of the distribution of the time difference between the signal in one OM and the signal given by the reference photodiode of the laser during the dark room calibration.

An example of the distribution of the difference between the time of the signal recorded by an OM and the emission time of the pulse as recorded by the laser photodiode is shown in Figure 8. The mean value of this distribution yields the difference between the laser light signal time measured by the PMT (including the PMT transit time and the electronics delay) and the time measured by the photodiode channel. The sigma of the distribution is $0.5 \mathrm{~ns}$.

The time offsets between each OM of a line and the first OM of its lowest storey, which is chosen as a reference, are computed for each of the 30 ARSs of each sector. An example result is shown in Figure 9. The spread of the time offsets is of the order of a few nanoseconds, and is due to differences in the internal cabling of the OMs to the storey electronics as well as differences in PMT transit times. These time offsets are stored in the ANTARES database and are used as initial time constants in order to analyse data immediately 
after the installation of the line on the sea bed.

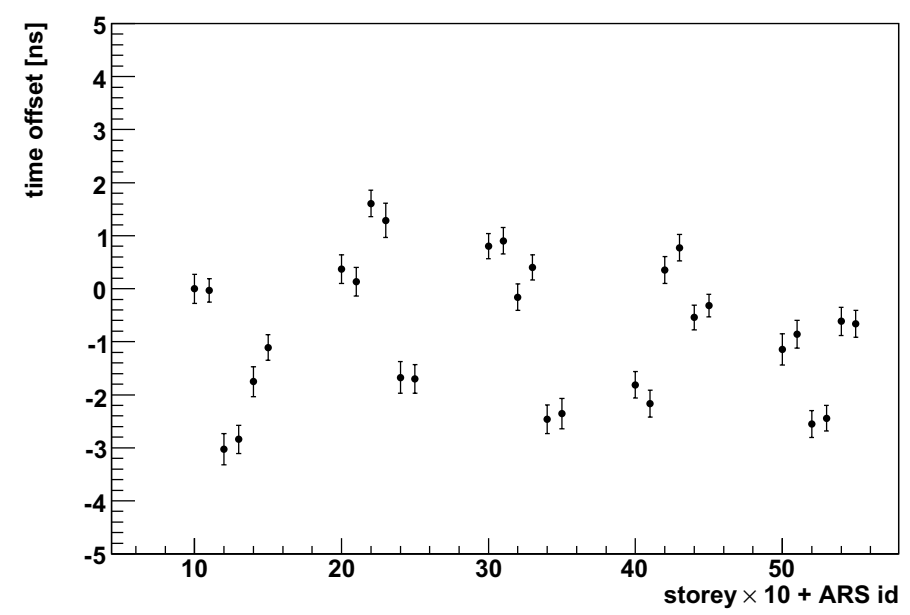

Fig. 9. Time offsets measured for all ARSs of one of the line sectors. The time offsets are relative to the first ARS of the first OM of the lowest storey. The ARS id runs from 0 to 5 within each storey.

The relative time calibration of the monitoring PMTs of the LED optical beacons (used for the in situ calibration described in subsection 4.2.1) is also performed during the onshore calibration. One of the fibres of the optical splitter is used in this case to send the light to the small PMT within each OB. Since this PMT is meant to measure the relatively high intensity of light emitted by the LEDs, the incident laser light intensity is tuned to be about twenty times higher than that provided to the OMs.

\subsection{In situ calibration}

The in situ measurement of the time offsets of all the OMs is performed with the optical beacon system [7]. This comprises two kinds of complementary devices: LED beacons that emit blue light $(\lambda=470 \mathrm{~nm})$ and laser beacons emitting green light $(\lambda=532 \mathrm{~nm}$ ) (see [9] for a description of the response of the $\mathrm{OM}$ as a function of the wavelength). Pictures of both devices before assembly are shown in Figure 10. The LED beacons are used to monitor the relative time offsets among OMs of the same line (intra-line calibration), whereas the laser beacons are used for monitoring the relative time offsets among the lines (inter-line calibration) and the calibration of the lowest storeys. The in situ calibration allows to re-measure the time constants computed onshore and is particularly important in case of a change in the PMT high-voltage and threshold settings. 

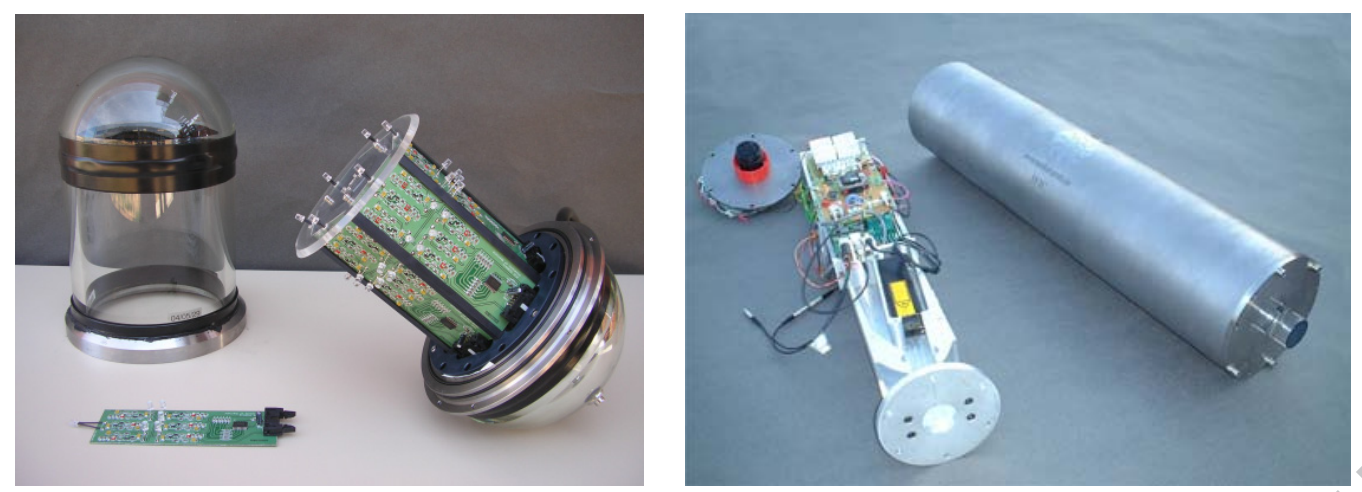

Fig. 10. Picture of an LED optical beacon (left) and a laser beacon (right).

\subsubsection{LED beacons}

Four LED optical beacons are located along every line of the detector in storeys 2, 9, 15 and 21 (counted from bottom to top). Each LED OB contains 36 LEDs distributed on six faces forming a hexagonal prism. The LEDs emit blue light with a maximum intensity of $\sim 160$ pJ per flash $\left(\sim 4 \times 10^{8}\right.$ photons per pulse) and a pulse width of $4 \mathrm{~ns}$ (FWHM). The emission time of the LED light is measured by an 8-mm PMT placed inside the frame of the LED OB (see reference [7] for more details).

Figure 11 shows the distribution of signal time residuals, i.e. the difference between the signal time recorded in the OM and the emission time of the corresponding LED flash, using the time offsets measured in the dark room and once the nominal travel time of the light from the beacon to the OM has been subtracted. The plot on the left shows the distribution corresponding to an OM close to the OB $(\sim 30 \mathrm{~m})$ which receives a high amount of light. The plot on the right shows the distribution for an OM located seven storeys above the $\mathrm{OB}(\sim 100 \mathrm{~m})$. In this case, a tail at positive times due to light scattering in water can be observed. A Gaussian fit to the region including the rising edge of the time distribution and the first bin after its maximum is performed. The range of the fit is restricted in order to use mostly the earliest photons, whose delay due to scattering can be neglected.

The position of the time residual peak, i.e. the mean value of the Gaussian best-fit curve described above, as a function of the distance from the OM to one optical beacon is shown in Figure 12, together with the slopes of such fits for all the OBs. The increase of the time residual with distance originates from an "early photon" effect, arising from the duration of the light pulse and the fact that the first photons detected by the PMT determine the recorded time of the pulse [13]. The PMTs closer to the beacon, which detect a higher amount of photons per pulse, tend to trigger on the photons emitted earlier in the flash, whilst for PMTs farther away the probability distribution for the measured time of the signal is determined by the pulse width. The arrival time of the first 

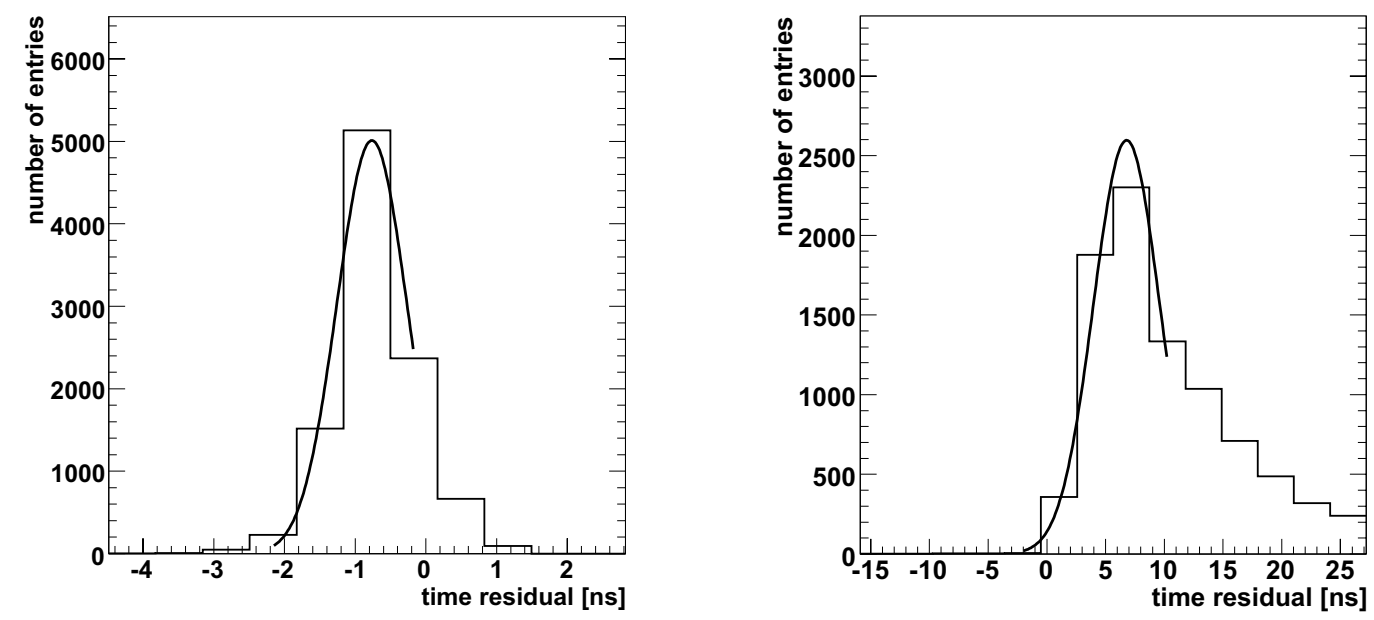

Fig. 11. Time residual distribution in two different OMs located two storeys (left) and seven storeys (right) above the LED flashing beacon. The curve is the Gaussian fit explained in the text.

photon obeys the order statistics [14]. A Monte Carlo simulation taking into account this effect as well as the exponential decrease of the intensity caused by the light attenuation shows that a linear increase in the time residual is expected when the number of photons reaching the PMTs is sufficiently high. A straight line is fitted to the time residual peaks ordered by the distance of the optical modules from the optical beacon. If a point deviates more than $2 \mathrm{~ns}$, the fit is redone excluding it. The deviations from the fit are then used to obtain the time offset correction. The distribution of these time offset corrections for all OMs that can be calibrated with the OBs is shown in Figure 13. It is found that $15 \%$ of the cases need a correction to the dark room offsets larger than $1 \mathrm{~ns}$.

Seven storeys above each OB, excluding the one just above the beacon (which receives too much light), can be calibrated. The storeys farther away, which do not receive enough light, are monitored with the next $\mathrm{OB}$ along the line. The OMs of the first three storeys of each line can not be calibrated with the LED OBs since they are below or just above the lowest OB. For these, the time offsets given by the dark room measurements are used, corrected by the laser beacon and potassium-40 measurements as explained later.

A way to check the validity of a set of time offsets is to calculate the signal time differences for pairs of OMs in the same storey (intra-storey time differences) when illuminated by the optical beacon. In Figure 14, the intra-storey time differences for one of the lines using the time offsets measured onshore and in situ are shown. The time differences become smaller when using the in situ offsets. For a detector configuration when 10 lines were installed, the RMS of the average intra-storey time difference distribution for the OMs which can 

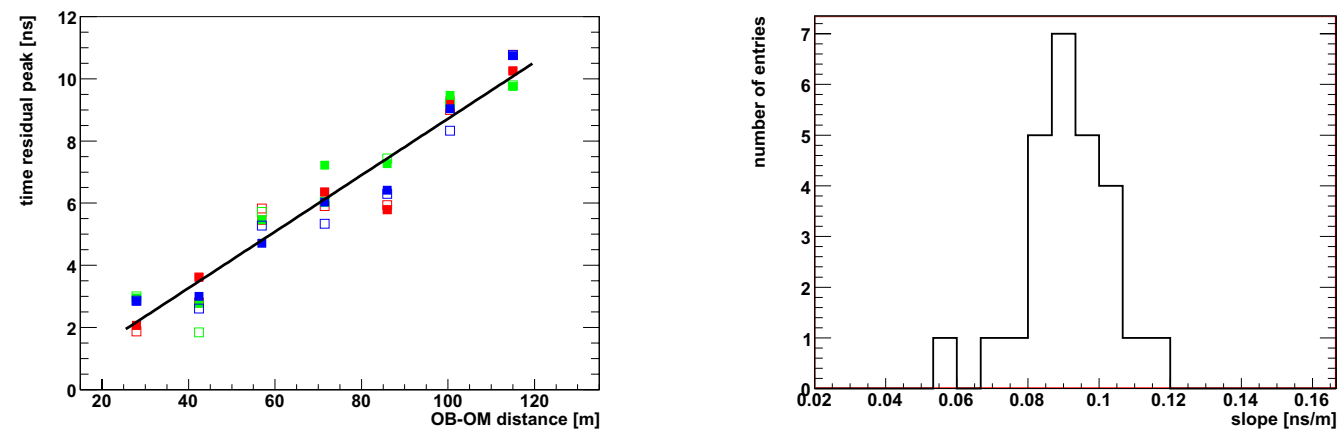

Fig. 12. Left: Time residual peak position as a function of the OB-OM distance, i.e. the distance between the optical beacon and the different optical modules, for all the ARSs along seven storeys. The six points at each distance correspond to the six ARSs in each storey (two ARSs per OM). The additional delay with distance is due to the early photon effect (see text). Right: Distribution of the slopes of the fits to the time residual peak positions as a function of the OB-OM distance for all the OBs.

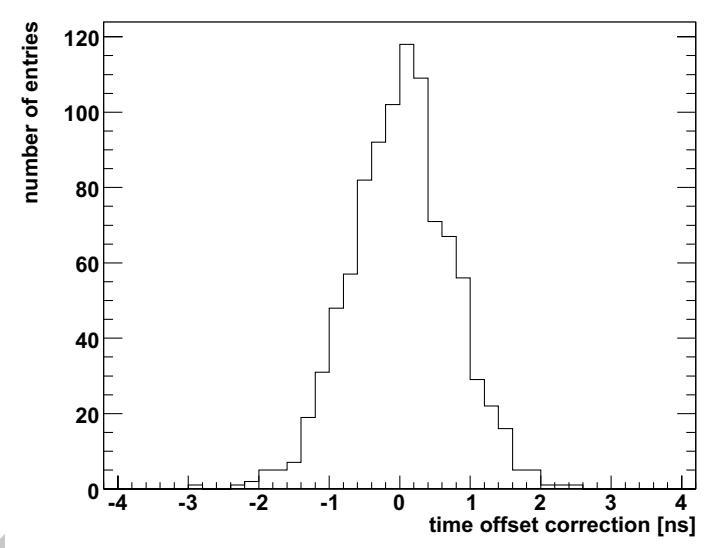

Fig. 13. Distribution of the time offset corrections measured in situ with the LED optical beacons. Each entry is calculated as the difference between the time residual peak shown in Figure 12 and the corresponding value of the fitted line.

be re-calibrated decreases from 0.72 ns to 0.48 ns after the application of the in situ calibration. This is a $30 \%$ improvement, which is equivalent to a subtraction of $0.54 \mathrm{~ns}$ in quadrature. In terms of sigma, the change is from $0.60 \mathrm{~ns}$ to $0.33 \mathrm{~ns}$. Initially calibration runs were performed once per week, but after the time offsets were found to be very stable, the frequency has been reduced to once per month. Since each set of calibration runs takes about two hours, the total dead time is about one day per year, which is considered short enough. 

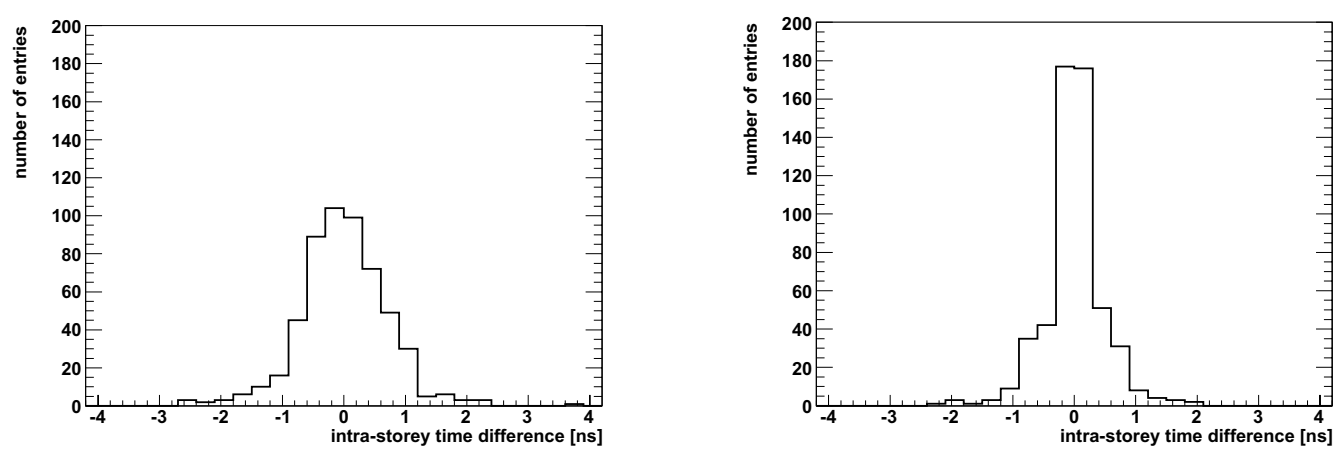

Fig. 14. Time differences between OMs in the same storey for one of the lines using the time offsets measured onshore (left) and in situ (right).

\subsubsection{Laser beacon}

The relative time offsets among lines can be obtained with the laser beacons. This inter-line calibration is needed because the time offsets measured in the dark room are calculated with respect to a reference OM specific to each line. Being much more powerful than the LED beacons, the lasers can illuminate all detector lines. Given that only distances where the OMs are illuminated below the one-photoelectron level are considered and since the time width of the laser pulse is very narrow (FWHM $\sim 0.8 \mathrm{~ns}$ ), the early photon effect is negligible. As a consequence, the time residuals do not depend on the distance to the source, as can be seen in Figure 15. The relative time offsets among lines are then computed as the average of the time residual peaks. The calculated inter-line time offsets are shown in Figure 16. In addition to the inter-line calibration, the laser beacon also provides a tool to compute the time offsets of the OMs in the lowest storeys.

\subsection{Potassium-40}

The radioactive potassium-40 $\left({ }^{40} \mathrm{~K}\right)$ present in sea water can be used for time and efficiency calibration of the detector using the Cherenkov light induced by the electron emitted in the $\beta$-decay of potassium $[3,15]$. If such a decay occurs within a distance of a few meters from a storey, coincident signals can be recorded by two OMs. In Figure 17 (left) an example of the distribution of the measured time differences between hits in two OMs of the same storey is shown. A clear peak is found, in good agreement with the expectations from simulations. The data has been fitted to the sum of a Gaussian distribution and a flat background. The width of these distributions (FWHM=9 ns) is set by the difference in the distance from the point where the decay occurs to each of the OMs of the pair. The position of the peak can be used to cross-check within each storey the time offsets provided by the onshore dark room and optical 


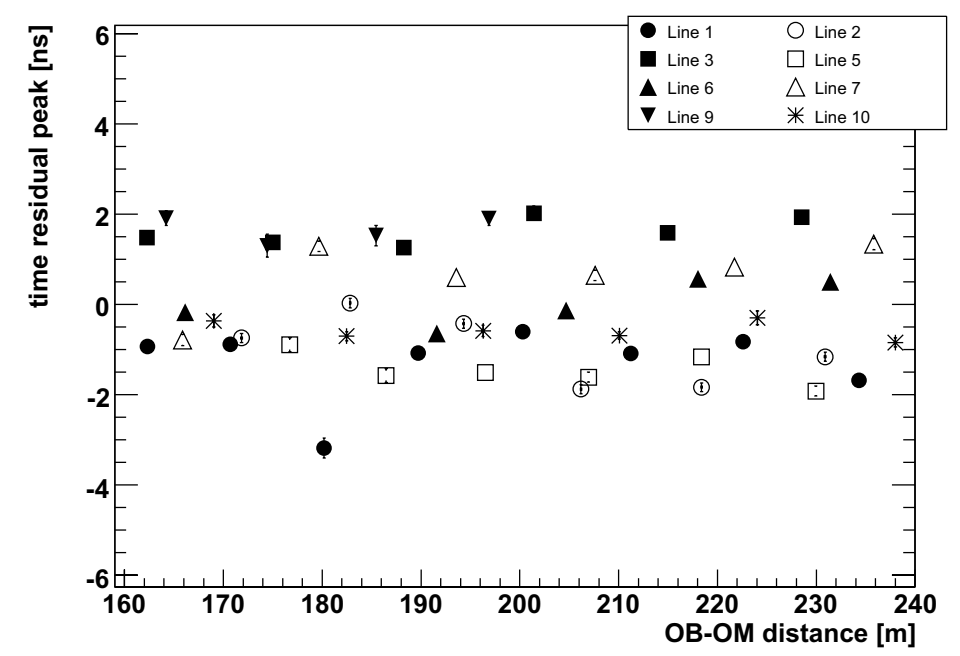

Fig. 15. Time residual peak position versus the distance between the laser beacon and the OM. Each point is the average over all the OMs in the same storey.

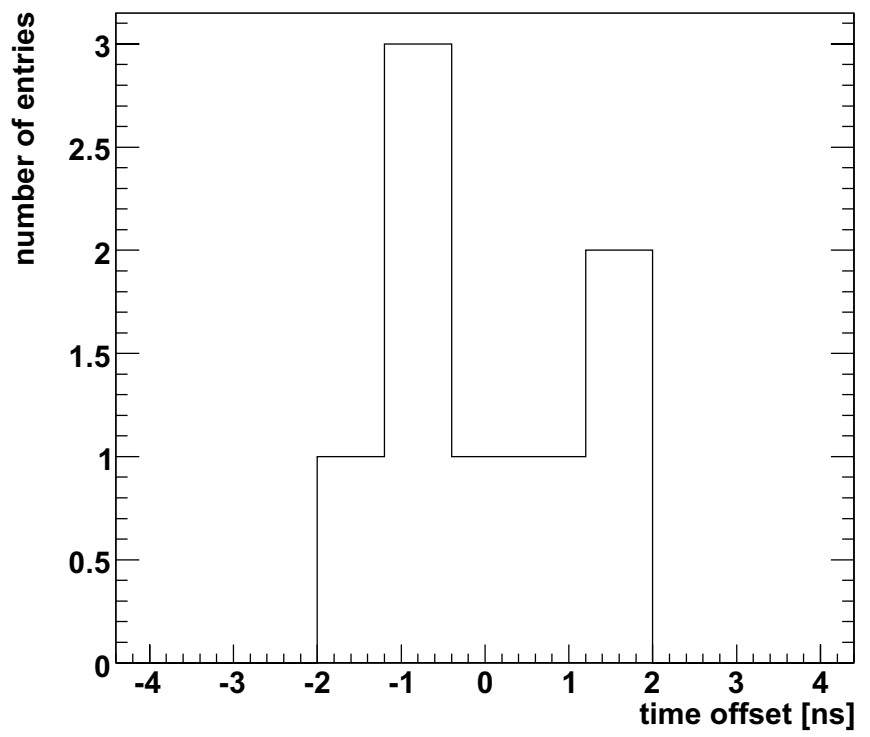

Fig. 16. Distribution of the inter-line offsets calculated from the measurements made with the laser beacon. Each offset is calculated as the average of the time residual peaks shown in Figure 15.

beacon calibrations (Figure 17, right). If the time offset of one of the OMs of the pair were incorrect, we would see that the peak is displaced from zero. The RMS of the mean intra-storey time difference distribution determined by the ${ }^{40} \mathrm{~K}$ improves from $0.72 \mathrm{~ns}$ to $0.57 \mathrm{~ns}$ when using the time offsets calculated in situ rather than those determined from the dark room calibration. It is worth noticing that the ${ }^{40} \mathrm{~K}$ intra-storey calibration is independent of the LED OB system and relies on a completely different light source: the ${ }^{40} \mathrm{~K}$ is a dim, 


\section{ACCEPTED MANUSCRIPT}
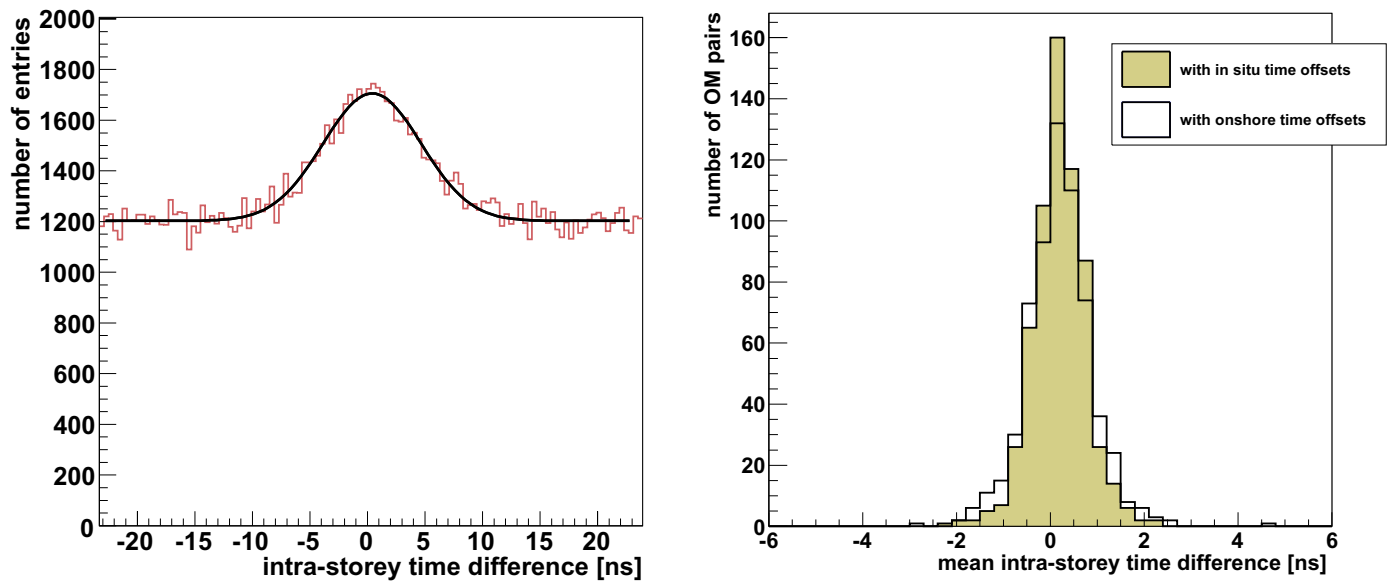

Fig. 17. Left: Distribution of background hit time differences for one pair of OMs in the same storey. The peak is due to single ${ }^{40} \mathrm{~K}$ decays detected in coincidence by two OMs. The data have been fitted to a sum of a Gaussian distribution and a flat background from random coincidences. Right: Comparison of the distributions of mean intra-storey time differences from ${ }^{40} \mathrm{~K}$ using the onshore and in situ time offsets.

distributed and closeby source, whereas the beacons are powerful, point-like and distant sources.

\subsection{Internal LEDs}

Each optical module also incorporates an internal LED (of the same model as those used in the LED beacons) which is used exclusively to monitor the stability of the PMT transit time. The LED is located on the back of the phototube and illuminates the photocathode from behind. It is triggered by the clock signal at a constant rate. Figure 18 shows the mean time of the internal LED flashes as recorded by the corresponding PMT as a function of time. The values, measured in situ every week, vary less than 0.2 ns (RMS) over an eight month period.

\section{Time resolution of the front-end electronics}

The impact of the front-end electronics on the time resolution can be determined during onshore and in situ calibrations. In this section we present three different methods to estimate this front-end electronics contribution.

At low light intensity, the time resolution of an OM measured in the laboratory is dominated by the transit time spread of the PMT $\left(\sigma_{T T S} \sim 1.3 \mathrm{~ns}\right)$. At 


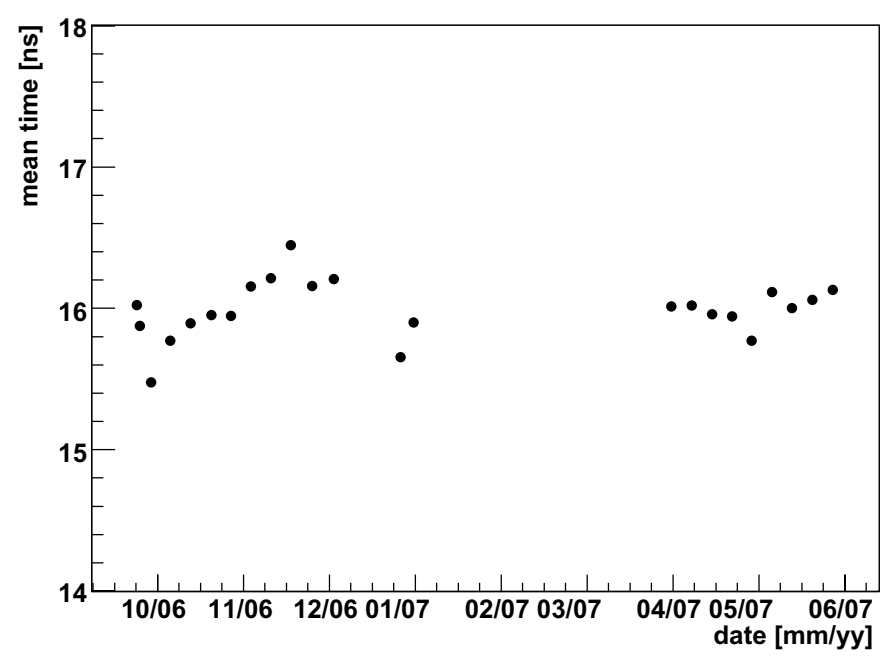

Fig. 18. Example of the mean time of the internal LED flashes as recorded by the $\mathrm{PMT}$ as a function of the time.

high intensity, this contribution decreases as the square root of the number of photoelectrons and therefore the dominant term to the width of this distribution is the constant contribution due to the front-end electronics. This irreducible contribution is found to be $\sim 0.5 \mathrm{~ns}$ in the dark room calibration (see Figure 8).

The in situ estimation of the time resolution of the electronics is obtained from the distribution of the difference in the time measured by an OM close to an $\mathrm{OB}$ with respect to the emission time of the OB pulse. The sigma of the time distribution of the signal in an $\mathrm{OM}$ is given by

$$
\sigma_{O M}^{2}=\frac{\sigma_{T T S}^{2}}{N_{p e}}+\frac{\sigma_{\text {water }}^{2}}{N_{\gamma}}+\sigma_{O B}^{2}+\sigma_{\text {elec }}^{2}
$$

where $\sigma_{T T S}$ is the transit time spread of the PMT, $\sigma_{w a t e r}$ is the spread due to the scattering and chromatic dispersion of light in water $(\sim 1.5 \mathrm{~ns}$ for a light path of $40 \mathrm{~m}), \sigma_{O B}$ is the uncertainty of the measured emission time of the pulse and $\sigma_{\text {elec }}$ the spread due to the electronics. For an OM close to an OB, $N_{p e}$ and $N_{\gamma}$ are high and their corresponding contributions become negligible. Due to the fast rise time of the internal PMT of the OB, $\sigma_{O B}$ can also be neglected. An example of the time difference distribution is given in Figure 19. Measurements with several OMs close to OBs give a time resolution of $\sim 0.5 \mathrm{~ns}$ for the electronics, in agreement with the results obtained during the onshore calibration.

A third way to check this time resolution is given by comparing the times measured by two OMs in the same storey when illuminated at high intensity. In 


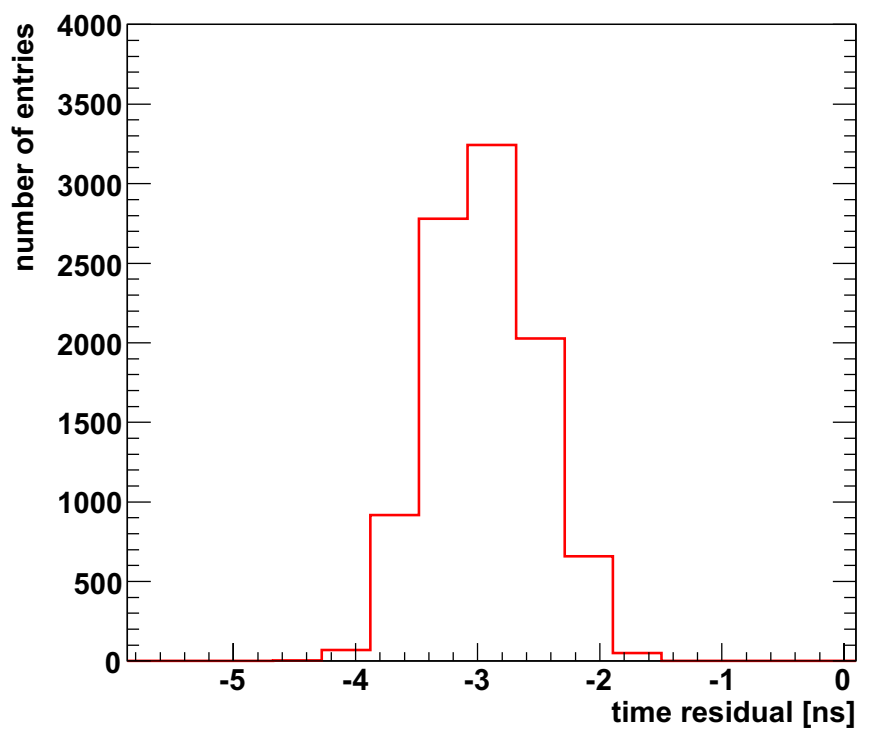

Fig. 19. Example of the time residual distribution for an $\mathrm{OM}$ located one storey above the optical beacon. The sigma of the distribution $(0.5 \mathrm{~ns})$ is dominated by the contribution from the front-end electronics.

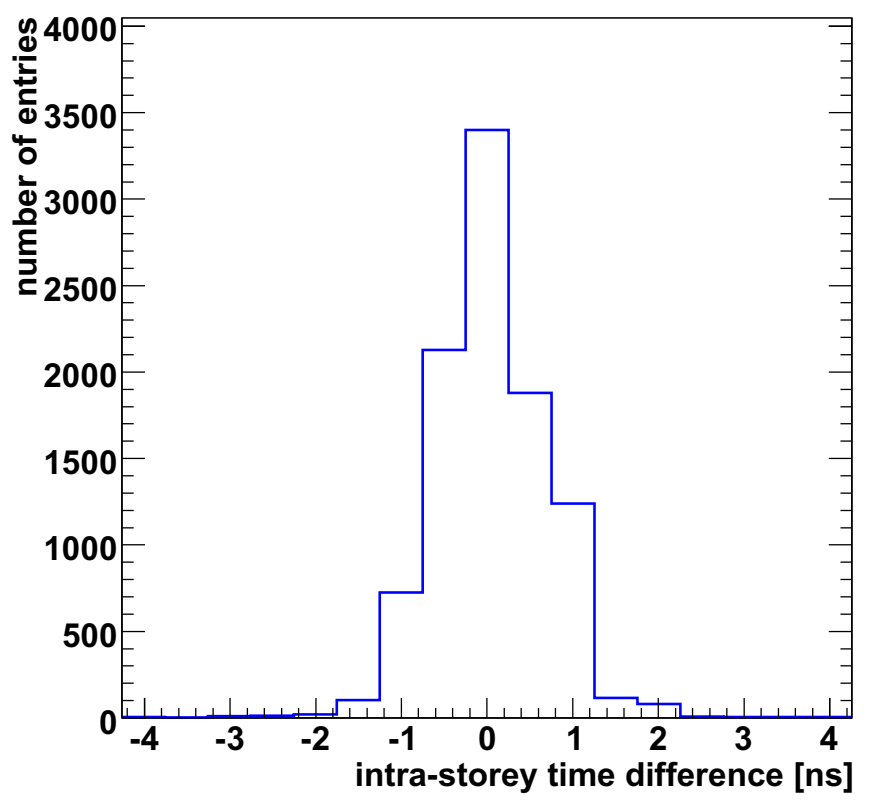

Fig. 20. Example of the distribution of the hit time differences for a pair of OMs in the same storey when illuminated by a close OB. The sigma of the distribution $(0.7 \mathrm{~ns})$ indicates a front-end electronics resolution of $0.7 / \sqrt{2}=0.5 \mathrm{~ns}$.

this case, the sigma of the distribution (see Figure 20) is given by $\sigma_{O M-O M}=$ $\sqrt{2} \sigma_{\text {elec }}$. A front-end electronics time resolution of $0.5 \mathrm{~ns}$ is obtained. 
These checks indicate that the uncertainty introduced by the readout system is negligible with respect to the irreducible contributions from the chromatic dispersion and TTS.

\section{Conclusion}

The completion of the ANTARES telescope has opened a new window to the neutrino Southern sky. Since the main physics goal of ANTARES is the search for cosmic point sources, an important feature is its angular resolution (a few tenths of a degree above $10 \mathrm{TeV}$ ), unsurpassed for this kind of instruments. The track reconstruction algorithms are based on the PDF of the photon arrival times to the PMTs. Therefore, in order to ensure an optimal performance, a precise time calibration of the detection system is crucial.

An onshore calibration performed in the laboratory provides a preliminary time calibration. Once the detector is deployed in the sea, time calibrations are performed in situ with a system of optical beacons. Although most of the corrections to the onshore time offsets are small, there are $15 \%$ of cases where they are larger than 1 ns. The observation of coincident photons from the decay of ${ }^{40} \mathrm{~K}$ provides a completely independent verification for these measurements.

The adopted calibration systems and methods attain a relative time calibration between detector elements of less than $1 \mathrm{~ns}$, as required.

\section{Acknowledgments}

The authors acknowledge the financial support of the funding agencies: Centre National de la Recherche Scientifique (CNRS), Commissariat à l'énergie atomique et aux énergies alternatives (CEA), Agence National de la Recherche (ANR), Commission Européenne (FEDER fund and Marie Curie Program), Région Alsace (contrat CPER), Région Provence-Alpes-Côte d'Azur, Département du Var and Ville de La Seyne-sur-Mer, France; Bundesministerium für Bildung und Forschung (BMBF), Germany; Istituto Nazionale di Fisica Nucleare (INFN), Italy; Stichting voor Fundamenteel Onderzoek der Materie (FOM), Nederlandse organisatie voor Wetenschappelijk Onderzoek (NWO), the Netherlands; Council of the President of the Russian Federation for young scientists and leading scientific schools supporting grants, Russia; National Authority for Scientific Research (ANCS), Romania; Ministerio de Ciencia e Innovación (MICINN), Prometeo of Generalitat Valenciana (GVA) and MultiDark, Spain. We also acknowledge the technical support of Ifremer, AIM and 
Foselev Marine for the sea operation and the CC-IN2P3 for the computing facilities.

\section{References}

[1] P. Coyle for the ANTARES Collaboration, "The ANTARES deep-sea neutrino telescope: status and first results", Proc. of the 31st ICRC, Lodz (2009), eprint astro-ph/1002.0754

[2] M. Ageron et al., ANTARES Collaboration, "Performance of the First ANTARES Detector Line", Astropart. Phys., 31 (2009) 277, eprint astro$\mathrm{ph} / 1007.1777$

[3] J.A. Aguilar et al., ANTARES Collaboration, "Measurement of the atmospheric muon flux with a $4 \mathrm{GeV}$ threshold in the ANTARES neutrino telescope", Astropart. Phys., 33 (2010) 86, eprint astro-ph/0910.4843

[4] J.A. Aguilar et al., ANTARES Collaboration, "Zenith distribution and flux of atmospheric muons measured with the 5-line ANTARES detector", Astropart. Phys., 34 (2010) 179

[5] A. Heijboer, "Track reconstruction and point source searches with ANTARES", Ph.D. thesis, Univ. of Amsterdam (2004), http://antares.in2p3.fr/Publications

[6] A. Kouchner for the ANTARES Collaboration, "High energy neutrino astronomy", Proc. of the 22nd ECRS, Turku (2010)

[7] M. Ageron et al, ANTARES Collaboration, "The ANTARES Optical Beacon system", Nucl. Instr. and Meth. A 578 (2007) 498-509, eprint astro-ph/0703355

[8] J.A. Aguilar et al., ANTARES Collaboration, "Study of large hemispherical photomultiplier tubes for the ANTARES neutrino telescope", Nucl. Instr. and Meth. A 555 (2005) 132-141, eprint physics/0510031

[9] P. Amram et al., ANTARES Collaboration, "The ANTARES Optical Module", Nucl. Instr. and Meth. A 484 (2002) 369-383, eprint astro-ph/0112172

[10] J. A. Aguilar et al, ANTARES Collaboration, "The data acquisition system for the ANTARES neutrino telescope", Nucl. Instr. and Meth. A 570 (2007) 107-116, eprint astro-ph/0610029

[11] J. A. Aguilar et al, ANTARES Collaboration, "Performance of the front-end electronics of the ANTARES Neutrino Telescope", Nucl. Instr. and Meth. A (2010), doi:10.1016/j.nima.2010.06.225, eprint astro-ph/1007.2549v1

[12] JDS Uniphase Co., http://www.nanolase.com

[13] J. A. Aguilar "Analysis of the Optical Beacon system and search for point-like sources in the ANTARES neutrino telescope", Ph.D thesis, Univ. of Valencia (2007), http://antares.in2p3.fr/Publications 
[14] M. Kendall and A. Stuart, "The advanced theory of Statistics I", Ed. Oxford University Press, ISBN 0852642857

[15] P. Amram et al., ANTARES Collaboration, "Background light in potential sites for the ANTARES undersea neutrino telescope", Astropart. Phys. 13 (2000) 127-136, eprint astro-ph/9910170 